\title{
Preface for the thematic issue
}

\author{
Ben Jonker • Werner Schiehlen
}

Received: 3 August 2013 / Accepted: 3 August 2013 / Published online: 24 August 2013

(C) Springer Science+Business Media Dordrecht 2013

The Euromech Colloquium 524 on multibody system modeling, control and simulation for engineering design took place at the University of Twente, The Netherlands, on February 27-29, 2012. There were 41 participants from 9 European countries and 1 non-European country.

The Colloquium was devoted to multibody system dynamics with regard to engineering design principles, modeling for design and simulation. A broad variety of engineering applications have been presented from nano motion systems to robotics and biomechanics as well as vehicle dynamics up to historical masonries in civil engineering. Both detailed analyses using large scaled industrial models as well as high level analyses based on simple (prototype) models with a small number of degrees of freedom were presented.

The application oriented sessions clearly demonstrated that multibody system modeling techniques can effectively support the different phases of the design and optimization process, mainly because of their computational efficiency and their ability to allow models to be easily updated and extended during the design process. Obvious key aspects in this context appeared to be the development of efficient formulations and numerical integration techniques. In case of flexible multibody systems model reduction techniques are of importance. In various sessions there were presentations that demonstrated the importance of the development of contact algorithms.

Based on recommendations of the members of the Scientific Committee, some selected authors have been invited to submit their contributions as peer-reviewed papers in this Thematic Issue of the journal Multibody System Dynamics.

We wish you a most interesting reading.

B. Jonker

Faculty of Engineering Technology, University of Twente, P.O. Box 217, 7500 AE, Enschede, The Netherlands

e-mail: J.B.Jonker@utwente.nl

W. Schiehlen $(\varangle)$

Institute of Engineering and Computational Mechanics, University of Stuttgart, Pfaffenwaldring 9, 70569, Stuttgart, Germany

e-mail: Werner.Schiehlen@itm.uni-stuttgart.de 